\title{
Facts and Anomalies to Keep in Perspective When Designing an Artificial Intelligence
}

\author{
Navin K Ipe
}

\begin{abstract}
The design of any artificial intelligence is an interdisciplinary pursuit that requires keeping within perspective, the various properties of matter and life in the known universe, while remaining cautious of biases and misconceptions that arise from the limitations of prior learning, available tools and sensory capabilities. This paper curates a vast collection of human knowledge gathered during the process of exploring and questioning the fundamentals of why the mechanisms that constitute life were built in specific ways, and re-questioning those facts using anomalies that sometimes contradict or expose errors in human assumptions of life and intelligence. A meta-analysis of such knowledge at a single glance also helps identify interesting patterns in various phenomena that can in-time, spur creative solutions, alter the direction of research, assist with intelligent inferences and hopefully result in the identification and creation of artificial intelligence.
\end{abstract}

Index Terms-artificial intelligence algorithmic design and analysis, artificial general intelligence, cognitive agents, machine intelligence, machine learning

\section{INTRODUCTION}

$\mathbf{S}$ CIENTIFIC research consists of vast labyrinths of human knowledge, built by the toil of researchers who have attempted uncovering the secrets of the universe. Standing on the shoulders of scientific giants, this paper presents a preliminary meta-analysis of a very wide perspective of facts and anomalies that need to be kept in mind when conceptualizing the design of any Artificial Intelligence (AI).

The discovery of wavelengths beyond the human-audible and human-visible spectrum, heliocentrism and the properties of quantum physics are some examples of how new knowledge was created with keen observation and logical deductions, despite the biases and limitations of contemporary knowledge, sensory inputs and available tools. It would be wise to learn from the innocent errors of reputed philosophers like Aristotle, who assumed that flies originated from putrid matter, and crocodiles were created from rotting logs [1]. Intelligence is currently known as the ability to infer information, retain it and apply it adaptively in an environment [2]. However, since the constitution of intelligence is unknown, and pursuits to simulate general intelligence are yet to achieve success, it helps to re-evaluate the direction that research is taking, and thinkthrough the corrective steps necessary to channel existing research in a direction that is more likely to achieve objectives. This paper deviates from conventional styles of presenting

Author acknowledges the support received from M S Ramaiah University of Applied Sciences, Bengaluru, India. N. K. Ipe is with the Department of Computer Science and Engineering, M S Ramaiah University of Applied Sciences, Bengaluru, India (e-mail: navinipe@gmail.com).

Manuscript received May 16, 2020 information, to assimilate a broad spectrum of fascinating knowledge, analyze it, utilize it to rule out misconceptions and derive insights from anomalies and observed patterns. The objective is not to define intelligence, but to help minds escape biases created by perceptions of intelligence and to trigger new ideas for the architecture and design of both the technology for AI and for innovative biological experimentation that could result in new discoveries. AI history is scattered with repeated hopes and claims [3] of being at the threshold of creating a viable AI, followed by disappointment and scathing reviews. Some researchers have proposed that AI needs to be built from the ground-up [4], and building a true AI does indeed require identifying the basic characteristics of intelligence, analyzing anomalies and associating them with similar phenomena that offer clues about its architecture and purpose. The observations presented in this paper are not meant to be exhaustive, but sufficiently broad in scope and reasonably concise, to keep in perspective various phenomena that need to be accounted for, when designing any AI. The list of information presented in this paper is a first step. It is to be followed by a systematic effort at utilizing this information to explore further and generate hypotheses that logically narrow down and identify the core principles that constitute intelligence.

Section II presents similar research that has investigated the basis of intelligence, Sect. III presents knowledge of various fascinating facts and anomalies that tingle the mind and help narrow down on what constitutes intelligence, Sect. IV] presents inferences derived from Sect. III and the paper concludes with Sect. V

\section{RELATED WORK}

There appears to be no other work that has attempted assimilating such a vast spectrum of knowledge, but history is witness to a swathe of persistent efforts at creating intelligent machines and examining the nuances of biological life. This section presents a small subset of such efforts. Ancient Greek myths spawned ideas like Talos, which were among the first documented AI conceptualizations. By the $17^{\text {th }}$ century, Rene Descartes and contemporary anatomists who were influenced by the concept of mechanical statues and clocks, proposed theories of the body being similar to a machine, and theorized that ideas originated from both sensory experiences as well as from the conscious mind. In 1747, a French physician La Mettrie concluded from observations, that "The human body is a machine that winds up its own springs; it is a living image of the perpetual motion”. In 1854, George Boole presented Boolean algebra as the mathematics of human intellect [5], 
but it was only until the 1940's that abstract reasoning by machines was made possible with the Church-Turing thesis and the invention of the digital computer which demonstrated that any mathematical operation could be performed using 0 and 1. Research into AI and its christening began at Dartmouth College in 1956 [6], where scientists from various fields attempted precisely describing every aspect of intelligence, to simulate it via a machine, followed by other scholars who pondered deeply about life [7]. Other researchers like David Marr rejected symbolic approaches [3] and stressed on the importance of AI understanding concepts from the bottom up. The AI is to be allowed to possess sensorimotor skills which would eventually help it build commonsense. Researchers also agreed with Moravec's paradox, that abstract reasoning was among the least important skills for an AI to possess, as detailed in the paper "Elephants Don't Play Chess" [8], where the author argues that "the world is its own best model". Cognitive scientists took the concept forward via the embodied mind thesis [9]. Post-2011, work on AI has focused predominantly on Computational Intelligence (CI), deep learning and big-data, where Convolutional Neural Networks (CNN's) and Recurrent Neural Network's (RNN's) have bettered even human accuracy on recognition of handwritten digits, beat humans at answering general trivia questions and even games like Go and Poker [10]. However, the algorithms continued suffering from over-fitting, the vanishing gradient problem and persistent criticism about the definition and method of evaluating intelligence being fundamentally flawed [11] [12], due to important properties of the brain's cortex being ignored [13]. Although attempts have been made to map the flow of brain activity to determine the relationship between the sensory and cognitive operations in various cortexes [14] and a more recent study on the biological basis for intelligence has identified genes and cellular properties associated with intelligence [15], the fundamental secrets of intelligence are yet to be unlocked.

\section{OBSERVATIONS}

Various past inventions and discoveries were the result of comparing similar patterns in phenomena, paying attention to anomalies and investigating deeper. Although the field of AI has undergone such scrutiny, it is imperative to keep in mind many more realities of the universe and details of life, before making assumptions about intelligence or designing intelligent machines. This section lists many such realities presented as disparate entry-points into important facets of knowledge that constitute life. A quick-read through this section can help eliminate biases and trigger ideas in the mind of the reader, hopefully leading to greater inquisitiveness and discoveries.

\section{A. The universe}

Limited perspective once led people to believe that the Earth was at the center of the universe [16] and humans were the most important of all creation. It was the observations of Nicolaus Copernicus, Galileo Galilei, the Big Bang theory proposal and many other scientific observations that eventually altered opinions and brought forward theories that the universe began approximately 13.8 billion years ago and may be 93 billion light years wide. The Milky Way galaxy itself contains around 200 billion stars and the universe consists of vast expanses of dark matter, dark energy, ordinary matter, antimatter and electromagnetic radiation [17]. An important pattern to note here is that the forces or laws of nature acting on matter depend on the properties of matter at their respective scales. At large scales, conventional theories of gravity and relativity are unable to explain the rotation of galaxies [18] and at small scales, it is theorized that bacteria may be unable to detect gravity [19] due to their small size.

1) Anomalies: Life exists on a seemingly insignificant speck of matter (Earth) in a universe that appears to contain no other form of life or activity in large volumes of its vast expanse, but may potentially harbor life in denser areas [20]. Similarly, microbes exist on specks of matter that are insignificant in size compared to the volume of the Earth. In a universe that harbors various forms of activity (wind, revolutions, nuclear reactions, nebulae, supernovas, comets, black holes etc.), where known and unknown forces give planets, stars and galaxies certain shapes, the pattern of life is an anomaly due to living agents possessing active bodies and highly adaptive, social, directed behaviors (including microbial intelligence [21]), memories that are capable of accounting for past phenomena, chemical properties and anticipating future phenomena.

\section{B. Life on Earth}

Earth's biodiversity is evidence of how multiple independent agents can gradually discover properties of their environment and use specialized technology in their bodies to take advantage of what the environment offers. Even more astounding, is the generalization capability of a storage mechanism like deoxyribonucleic acid (DNA) and ribonucleic acid (RNA), which encode a vast array of data. The Earth potentially contains one trillion species, each specializing in incredible feats of technology, as listed in Table I. Science is yet to ascertain how birds and fishes discovered they could utilize the Earth's magnetic field to determine direction, how ants and other species realize that the weather is going to turn rough, how plants and bacteria designed seeds and spores which allow them to survive for centuries, how slugs achieved self decapitation [22], how clams realized that calcium could be utilized to create a shell, how cone snails discovered that fish could be sent into hypoglycemic shock using insulin [23], what kind of a decision process allowed whales to evolve such a huge body full of cells that coordinate so well, how creatures developed eusocial behavior, how insects learnt to make sense of visual inputs from multi-faceted ommatidium, how the green-banded broodsac learnt to manipulate behavior in hosts [24], how a snake evolved a tail that looked like a spider, to lure birds [25], how the Warnowiid dinoflagellate acquired what appears to be an eye with a lens, how the Macropinna microstoma evolved a transparent head that protects its eyes [26], how snails created chemicals that constitute the slime which helps them move (and other snails that create metal armour from iron-rich environments [27]), how the Tardigrade 
used the Dsup protein in its DNA to protect the DNA from radiation (Dsup when applied on human cells also suppresses X-ray damage by $40 \%$ [28]). The entire body of an organism adapts to fulfill the special ability it possesses, and it is interesting to note that no organism appears to possess the capability of integrating multiple such special abilities. For example, there is no chameleon which has the ability to spin a web, possess ommatidium, survive in space, survive lack of water, possess bioluminescence and also fly while echolocating prey.

1) Anomalies: The variety of capabilities listed in Table I challenge the assumptions based on which current algorithms are built. Consider:

- Prions appear to mutate and adapt without a need for nucleic acids [42].

- The multicellular sea sponge coordinates its bodyprocesses and possesses a long-term memory without utilizing a neural network [43].

- The Turritopsis dohrnii can reverse its biotic cycle to transform from adult stage to polyp stage.

- Humans are the only known creature capable of using technology at a rapid rate, to achieve a multitude of abilities without altering the human body to achieve the ability (wheels, x-rays, night vision, artificial light, flight, rocket propulsion, space-suits and now humans also aim to create intelligence).

\section{Details of life}

Simulating artificial life requires accepting the scale, complexity and equilibrium of real life. The following facts present a humbling perspective of how much knowledge is yet to be discovered and highlights the astounding level of technology that drives cellular life.

1) Purpose of life : Human knowledge is yet to bridge the explanatory gap [44] of noogenesis (creation of intelligence) and the purpose of life. Prior theories such as panspermia (life being abundant in the universe), life beginning from autocatalytic chemical reactions, theories based on chemicals and evidence from meteors, have attempted explaining [45] how the chemicals that constitute the building blocks of life may have come into existence, but Homo sapiens have existed on Earth a mere 300 kiloannums, compared to the 4.1 gigaannums that elapsed since life first appeared on Earth, and also compared to the 20 gigaannums since the theoretical "Big Bang". It is possible that events in the universe are unfolding too slowly and in too many dimensions for humans to perceive enough of it to make accurate conclusions.

2) Biological programming: Neurons are merely the tipof-the-iceberg in a multi-dimensional equilibrium of chemicals that constitute intelligence. In each cell, a biochemical cascade of events create cellular networks and communication mechanisms such as mechanotransduction, thermoception, osmosensors, ion channels, lipophilic messengers and redox signaling (intracrine signals), forming an integral part of the "biological software". DNA for example, is 2 nanometer in width, approximately 2 to 3 meter long and is packed so compactly using histones, that it occupies merely $10 \%$ of a cell's volume. The ACGTU nucleobases in DNA/RNA are arranged as triplets called codons, that specify the sequence of forming the gene regulatory network, protein synthesis, gene expression, gender determination, specification of the homeobox sequence (which specifies cell migration spatially and temporally within the embryo, to create distinct body parts), biochemical properties, behaviors and the shape of an organism (morphogenesis) [46]. The complex process of copying DNA is even more fascinating. A helicase molecule breaks hydrogen bonds to "unzip" DNA before replication is initiated. During replication, one error can occur per 100000 nucleotides, which can add up to 120000 errors in the 6 billion base pairs [47]. DNA polymerase detects and corrects replication errors to maintain fidelity of the original DNA strand. The replication is performed so faithfully that retroviruses can insert viral DNA into infected cells, and the cell replicates the viral DNA. Currently, science possesses knowledge of only the mechanisms that occur, but the method by which these complex molecules follow a detailed procedure is unknown. Another interesting directed mechanism is the manner in which guidance proteins (semaphorin, ephrin, netrin, Slits) guide neurons in the brain [48] to form connections with specific neurons that are identified by molecular labels.

3) Genetic anomalies:

- Mutations in the Drosophila labial gene can cause the mouth and head structures that initially grow outside the body, to not get internally involuted, thus preventing the salivary gland and pharynx from growing [49].

- DNA not only stores information, but is also used to trap microbes by wrapping around it as a neutrophil extracellular trap [50].

- Normally, the genetic code is fixed in organisms, but some microorganisms are capable of dynamically increasing their genetic code [51].

- DNA can be artificially synthesized without a template DNA [52].

- Viruses exploit the molecular machinery in cells to "walk" on micro-tubules and to replicate themselves [53].

- Amoeba dubia has a genome size of 670 billion base pairs, but a human genome has only 2.9 billion base pairs [54].

- Certain trees like the Banyan and Pando [55] spread roots, forming a single genetic individual that appears to live forever.

- Transposons of DNA (called "selfish DNA") demonstrate that cells or genes could potentially exercise a choice about DNA transferability and its position in the genome [56].

- Without laboratory equipment, an isolated human will be unable to reproduce.

4) Biological communication: Cells recognize and communicate with each other (thus exhibiting social behavior [57]) via either mechanical forces detected or exerted by the cell, or via biochemical molecules which include autocrine, juxtacrine, paracrine and endocrine signals [58]. Surprisingly, even microbes in the human intestine communicate with the intestine's epithelial cells [59] [60]. A fascinating method 
TABLE I

SPECIAL ABILITIES OF ORGANISMS / AGENTS

\begin{tabular}{|c|c|}
\hline Organism/agent & Special abilities \\
\hline Infectious prion & Evolution without nucleic acids [29] \\
\hline Magnetospirillum & Produces magnetite from ingested iron and moves according to the magnetic field of the Earth (magnetotaxis) [30] \\
\hline D. radiodurans & Capability to repair its DNA even when damaged by 5000 grays of radiation 33$]$ \\
\hline T. gondii & Alters host organism's behavior via epigenetic re-modeling to make the host more susceptible to being preyed upon $[34]$ \\
\hline Slime molds & $\begin{array}{l}\text { Asexual reproduction, learning and habituation to environment without neurons (primitive cognition) 35], transfer of learning to } \\
\text { other cells via cellular fusion [36] }\end{array}$ \\
\hline Antlions & Digs a low-traction funnel in mud, uses venom, uses body waste to spin silk for cocoon, metamorphosis \\
\hline Termites & Digests wood, cooperatively designs, plans and builds a termite hill architecture \\
\hline Flies & Possess compound ommatidium, flight, sensitive antennae, agility, adhesive grip \\
\hline Sea Sponges & Re-mold bodies without using neurons, trap food by redirecting water flow, swim during larval stage \\
\hline Butterflies & Metamorphosis, flight, locating nectar, specialized proboscis, ultraviolet vision \\
\hline Tardigrades & Extreme resilience to temperature, pressure, dehydration, toxins, radiation, outer-space survival \\
\hline Axolotls & Amphibious, capability to regenerate almost any part of its body \\
\hline Angler fishes & Bioluminescence from bacteria, survival under high water pressure, males permanently fusing bodies with females $[\overline{39}]$ \\
\hline Frogs/Toads & Sticky saliva on long tongue to trap insects, detects earthquakes early, tunes ears to specific frequency, uses anti-freeze 40$]$ \\
\hline Salamanders & Regenerates lost limbs, amphibious, long tongue to trap prey, venom, camouflage, batesian mimicry \\
\hline Turritopsis dohrnii & Reversing its lifecycle via cellular transdifferentiation \\
\hline Tailor birds & Flight, building a nest by sewing leaves, laying eggs, ultraviolet vision \\
\hline Lyre birds & Flight, accurate mimicry of various sounds using a complex syrinx \\
\hline Venus flytraps & Acquires nutrition from soil, by photosynthesis and by trapping insects \\
\hline Octopuses & Camouflage, venom, intelligence to use tools, mimics shapes, possesses suckers on limbs \\
\hline Snakes & Motility without legs, venom, infrared sense, gliding in air, swimming, spitting venom \\
\hline Eels & Generates electric shocks of upto $860 \mathrm{~V}$, 41 , swimming, breathing underwater with gills \\
\hline Sharks & Electroreception, sleeps while swimming, parthenogenesis \\
\hline Redwood trees & Grows upto $106 \mathrm{~m}$ high, lives more than 2000 years \\
\hline
\end{tabular}

of information sharing also exists in the form of mobile genetic elements (plasmids) in bacteria that can be shared between bacterial cells. Communication requirements have also caused neurons to take specialized forms such as unipolar, bipolar, multipolar, anaxonic, pseudounipolar, basket cells, purkinje cells, Lugaro cells, spindle cells and more. Similar to cellular communication, multicellular organisms also possess autocommunication (communication sent and received by self) and various other modes of communication, which use organs as higher level sensors and receptors. Humans, as a biological organism, use specialized mechanisms like wired networks, wireless networks, satellite communication and even signals to contact potential extraterrestrial life.

5) The protoplasm and biological machinery: The majority of a cell's volume is water, but apart from the various organelles, lipids and plastids (in plants), an intracellular fluid called cytosol forms a complex gradient of various molecules whose functions are not fully known. Cells also possess a flexible cytoskeleton consisting of filaments and microtubules that gives shape to cells, allows cells to deform, helps perform muscle contraction and is even suspected to form a vibratory and conductive means of communication which is suspected to play a role in quantum neuronal information processing and consciousness (controversial) [61]. Cells also use machinery like adenosine triphosphate synthase, which is composed of sub-units that function as a rotor and axle, thus forming a nano-motor that synthesizes ATP, which is used to store and transport electrical energy. An incredibly precise mechanism is designed to harness energy from redox reactions and pump protons [62]. Various types of myosin and kinesin also perform functions of "walking" on cellular filaments to transport organelles larger than $20 \mathrm{~nm}$ within the cell. Other molecular machines like ribosomes, synthesize proteins, polymerases and spliceosomes that act on DNA/RNA.

6) Cellular anomalies:

- Cells are always generated from cells. There has been no documented evidence of a cell assembling another form of life external to itself (although there have been successful laboratory attempts at creating artificial cells that perform a limited function like blood filtration [63]). Even artificially produced meat is grown from real cells.

- While stem cells possess a pluripotency that allows them to differentiate into various cell types, certain cell-types like neurons do not undergo cell division due to a lack of centrioles.

- When considering why proteins do not get tangled up, the unexpected discovery of unstructured proteins being responsible for signaling and regulatory functions [64 were considered as anomalies, but it brought to light the multi-functional role of molecules when they exist in various shapes.

- Prokaryotes do not possess a distinct, membrane-bound nucleus.

- The cells of plants did not evolve complex eyes, even 
though they are so dependent on sunlight. They are however suspected to have primitive vision-like capabilities [65].

7) Senescence and death: Aging is attributed to factors like an unstable genome, exhaustion of stem cells, malfunction of mitochondria, etcetera [66], resulting in death of an organism. One of the ways cells die, is via disassembly. A process called apoptosis is initiated, during which neighboring cells neatly remove dead cell parts (efferocytosis) without allowing various chemicals in the disintegrating cell to affect the surrounding tissues. The necessity of aging and death is still a mystery.

\section{Some human abilities}

Cellular operations occur in patterns that are in many ways similar to the pattern of operations in larger clusters of cells like the human body. It is important to notice the variety, the extent of cellular cooperation and biological engineering involved in seemingly simple mechanisms, while also keeping in mind the knowledge arguments [67] and the reality that the understanding of the universe we possess is a function of our senses.

1) Hearing: Air pressure vibrates the eardrum and ossicles, causing a pressure differential in cochlear fluids that is transmitted across the basilar membrane. The cochlea distributes each Fourier component of sound to stimulate hair cells, where the shearing motion between the reticular lamina and tectorial membrane deflects hair to create mechano-electrical transduction, which is processed as sound in the auditory system. Audio is processed by the temporal lobe which also recognizes and perceives memory (music evokes past memories). The manner in which the brain processes audio can also cause auditory illusions like hearing conversations in random noises [68], and the brain can even distinguish between minor variations in frequency, as demonstrated by visually impaired people who navigate by performing clicking sounds using their tongue and listening to the reflected sound [69].

Music can induce cerebral blood flow in various portions of the brain, causing release of oxytocin, serotonin and dopamine, indicating linkage to reward, motivation, emotion and arousal, while unpleasant melodies can trigger the cingulate cortex, indicating emotional pain and conflict [70].

2) Vision: Prior to light-rays hitting the rod cells and cone cells of the human eye (where the cell membrane potential is altered by the absorption of photons by photoreceptor proteins), light passes through multiple transparent layers of nerves. Signals triggered on various such 150 million receptors are channeled via a mere 1 million nerves in the optic nerve, indicating potential visual data generalization, specialized encoding or pre-processing that reduces data dimensionality. Visual information is then processed by the visual cortex in the brain. A long-standing philosophical debate called the Molyneux problem (the question of whether a congenitally blind person whose vision is restored would recognize shapes by sight if the person was first allowed to touch those objects without viewing them) was resolved when it was noticed that congenitally blind people whose vision was restored, found it very hard to understand spatial information conveyed by sight.
They looked at streetlights outside a window and said the light was pasted onto the window glass. If a person walked away from them, they said the person was shrinking. When using tools, they preferred to close their eyes. [71]. One such person who did not recognize a lathe when he saw one, proceeded to close his eyes and touch the parts of the lathe. Then he opened his eyes and remarked: "Now that I have felt it, I can see" [72]. This clearly shows that senses form experiences as a combination of multiple sensory inputs. Visual anomalies [73] and observations that constitute the Gestalt principles of grouping (proximity, similarity, closure, good continuation, common fate, good form) [74] offer more clues into how vision is processed.

3) Touch, smell and taste: Human skin can have upto 500 mechanoreceptors of various kinds per $\mathrm{cm}^{3}$, which convey the sensation of touch to the somatosensory cortex in the brain.

The olfactory system ensures that odor molecules which dissolve into the mucus of the nasal cavity are detected by olfactory neurons, based on the odor concentration and then transmitted to various parts of the brain.

The gustatory system (sense of taste) consists of approximately 5000 taste buds, each containing approximately 100 taste receptors onto which molecules bind. A mechanically activated TMEM63 channel allows sensing the texture of food [75].

\section{4) Anomalies:}

- Damage to the amygdala impairs emotion recognition in music [76].

- A person with retrograde and anteretrograde amnesia had his musical memory intact, was able to play the cello and could remember music from the past [77].

- When blanks are left in music, people activate deeper parts of the auditory cortex to fill the blanks [78]. Nothing is known yet about how music is stored in the brain, but the manner in which people who stutter can sing normally, offers clues into how this memory is accessed.

- In the famous case of Mr. Waterman, who retained motor control but lost the ability to perceive touch after a viral infection; he could not control any part of his body without looking at it. Not knowing where his arms and legs were, led to a floating sensation (perhaps the same that people experience in their dreams of falling). This shows a distinction between neurons responsible for motor skills versus neurons responsible for touch [79].

- Humans are aware of the relative location of body parts and objects relative to the body, via proprioception senses, as demonstrated by a fascinating case of a blind painter who painted impressive paintings of the world via mental representations that were created by tactile inputs [80].

- Chemicals like miraculin which by themselves have no sweet taste, are capable of inducing a sweet taste to sour tasting chemicals [81].

- Loss of the ability to smell (anosmia) is among the last senses to be lost with age, and loss of taste is rarely observed [82].

- Audiovisual speech can be perceived as a totally different syllable, as demonstrated by the McGurk effect [83]. 
5) Physiognomy and the homunculus: Why do creatures need a face, when signaling can be achieved via electricity, chemicals, sound, touch and vision? Humans take decisions based on their judgment of a person's facial appearance [84]. The high weightage the brain assigns to interpreting patterns as faces (as seen in pareidolia [85]) and the manner in which exaggerated or distorted portions of faces in caricatures [86] are distinctly identifiable, is evidence of a plasticity in communication between the various cells that identify shapes and associate them using various portions of the brain. Researchers have also identified a sensory homunculus, which represents a map of brain areas dedicated to sensory processing for different anatomical divisions of the body. Similarly, a motor homunculus represents a map of brain areas dedicated to motor processing for different anatomical divisions of the body [87].

6) Time perception and temporal illusions: The inference model of time states that events are inferred by association between the event and events whose date/time is known. This is handled by the cerebral cortex, basal ganglia and cerebellum [88]. Audio, tactile and vision signals are processed at varied speeds by neurons. The brain creates a unified representation from these signals by waiting for $1 / 10^{t h}$ of a second and retrospectively interprets the event [89]. This sometimes results in temporal illusions. It is theorized that time perception may be a low-level function, since rats whose cortexes were removed, were still capable of estimating time intervals of upto $40 \mathrm{~s}$ [90]. Certain chemicals are also known to distort time perception [91].

7) Memory encoding and recall: Although the exact process of encoding memories is unknown, it is experimentally known to be influenced by attention, emotion and sensory inputs encountered during an event. Each sensory input is processed by portions of the brain cortex, combined in the hippocampus, compared with older memories and stored [92]. To process direction and space, the brain has a special spatial representation system that uses place cells and grid cells [93]. Memory is successfully retrieved when brain activity resembles the activity performed during the time of learning, and memory can be updated during retrieval [92]. The brain also has the capability to compress the perceived time between voluntary action and sensory result via a phenomenon called postdiction.

8) Anomalies:

- Only humans have a chin [94].

- A stroke (cell death in parts of the brain) can cause aphasia (language disorders, loss of ability to understand and loss of reading/writing skills but does not affect intelligence), dysarthia (affects muscles used for speaking but does not affect the ability to select words to speak or to understand others) and dyspraxia (inability to move muscles in the correct sequence needed for clear speech). Stroke can also make facial expression stagnant, alter vision and hearing. It can affect short-term memory, mood and the ability to focus and swallow [95].

- In 1951, experiments held at McGill University attempted sensory deprivation on students (using goggles, gloves and earphones). Students deprived of sensory inputs, lost their ability to think properly and started having hallucinations [96].

- The alien hand syndrome causes people to perform actions with their hands without consciously controlling it. They do retain a sense of ownership of the hand. Lesions on the posterior parietal cortex (which simulates selfrecognition) results in an inability to recognize ones own body parts and movements [97].

- Partial removal of the hippocampus and parahippocampal cortices did not impair Henry Molaison's [98] intellect, but demonstrated the importance of associating past memories for inferences.

- The persistence of an engram (memory trace) even after erasing synaptic memories, may indicate that memories are not stored only in neuron synapses [99].

- Phineas Gage, whose brain suffered damage at the frontal lobe, retained his intellect [100].

- People with anteretrograde amnesia are unable to create new memories.

- Sleep deprivation results in death [101].

- Dolphins, whales, seals and some birds are capable of putting one hemisphere of their brains to sleep [102].

- Sleep-walking and highway hypnosis [103] reveal modes of incredibly accurate sub-conscious motor-control and memory access.

\section{E. Cognition and psychology}

1) Cognition and learning: Cognitive science is yet to make headway in definitively explaining the concept of self, free-will and consciousness. However, given the manner in which prions adapt, and viruses make use of cellular mechanisms, it does beg the question of whether cognition has a more fundamental origin. Insects as small as ants have responded to the mirror test [104], and the function of mirror neurons controversially depict a fundamental mechanism of identification of self and associating it with similar concepts of the external world [105]. Emotion is also related to cognition, and is tied to the limbic structures and the pre-frontal cortex of the brain [106] [107]. When learning to speak, human children begin with phonological errors (removing few letters of the word or altering it into other letters). They go through a cooing stage with vocalizations like "eh" or "heh" [108], then a holophrastic stage where they speak single words and answer questions, the telegraphic stage where the child expresses feelings in sentences and finally the multi-word stage. It is interesting to note how a child's imitation of words and actions are a generalized version of the actual word or action. This is a vital clue that the brain stores information by adding "low resolution" information repeatedly to finally achieve perfection. On the other hand, children kept in extreme social isolation or raised with animals during isolation, imitate animal behaviors. When brought out of isolation, they do exhibit the ability to learn via sign language or spoken language, which leads to the hypothesis that spoken language is innate to humans. They are also known to hoard items they like, form bonds with people they like, relate past events, link them with consequences and even introspect on whether 
events were caused by their own doing, even if it was not. Examination of their brain activity also led to the conclusion that there is a difference between cognition and language acquisition [109]. Children without intellectual impairment, perform better at theory-of-mind (ToM) tasks (understanding mental states of self and others) [110]. Concepts of fluid intelligence, crystallized intelligence and Piaget's observations of four stages of cognitive development in children [111] also offer clues about the constitution of intelligence.

2) Starvation: Known as the Minnesota starvation experiment [112], a group of volunteers were subjected to starvation for six months. Starvation caused them to become progressively silent, immobile and apathetic. Trivial incidents annoyed them. They complained of tiredness, muscle soreness, cramps, depression, dizziness, lack of ambition, moodiness, a feeling of being aged, loss of libido and they became sensitive to noise. Their visual acuity was decreased only a little at high illumination but their auditory acuity for every sound frequency improved and their intellect was unaffected. Psychologically, they developed an overwhelming preoccupation with food and became introverted.

3) Asch, Milgram and Stanford experiments: Solomon Asch conducted a conformity experiment where he noted that $75 \%$ of his test subjects decided to agree with a group's opinions, even if the group deliberately took wrong decisions, the individual knew it was wrong and the individual was under no pressure to agree with the group [113]. Similarly, the Milgram conformity experiment showed that volunteers from all walks of life were willing to give electric shocks to a person, just because an authority figure told them it was alright. Only 14 of the 40 people eventually dared to disobey, but the remaining 26 obeyed. This demonstrated that obedience is a psychological mechanism linking political purpose and individual action. It creates a binding factor which results in adherence to authority to such an extent that it overrides training in ethics, sympathy and moral conduct [114]. In the Stanford prison experiment, people who were granted authority, were willing to humiliate, create disparity and physically abuse the people they had authority over [115].

4) Anomalies:

- Certain people are born with an insensitivity to pain, due to mutations in the SCN9A gene [116].

- Deja vu is negatively correlated with age, and occurs more in people with a variety of experiences in life [117].

- The manner in which cognitive illusions, auditory hallucinations, psychosis, photosensitive epilepsy and druginduced-hallucinations manifest themselves, provide various clues into how the brain compensates for chemical imbalances or incomplete or fast-paced sensory inputs.

- Anosognosia patients lack the ability to identify deficits in their bodily function [118].

- The fact that humans think of how they would exist after they die, could provide clues to the physical basis of cognition.

- Dreams do not always follow a logical pattern, and this creative process of the brain is evident in how pleasure is derived from cartoons, jokes, poems and story-telling.
- In an experiment, people who used pennies for many years were unable to recall the exact details of a penny from memory [119].

- Animals do not ask questions [120].

\section{F. The machine dimension}

Computation using machines began with analog techniques, progressed to digital, hybrid of digital and analog [121], and now is at the threshold of quantum computing [122]. In the digital space, although octal, hexadecimal and ternary representations were possible, binary representation of data gained prominence due to the simplicity of representing on/off states with electricity, the capacity to encode English numerals and alphabets within 8 bits and the prominence of English being used for programming languages. This exposes a reality of hardware and software: computing has historically been optimized for hardware, software, calculations, English, speed of execution, latency, memory, the limitations of readability and representation. Although attempts at artificial life [123], artificial chemistry [124], artificial cells [125], molecular informatics, neuromorphic computing [126], AI accelerator hardware, cognitive architectures [127], symbolic AI, antilogic (scruffy), sub-symbolic, embodied intelligence, computational intelligence, soft-computing and statistical learning have been attempted, there exists the reality that the fundamental building-blocks of hardware and software may need to be re-designed from the perspective of representing a multi-dimensional world-model and intelligence. Software is currently stored as 0 or 1 on various kinds of volatile and non-volatile memory, transported between circuits via buses, signals are multiplexed, demultiplexed, processed in hardware registers, encoded as higher level packets, checked for CRC errors, interfaced with other hardware and software, and molded into whatever function humans wish the machine to perform. Machines are thus supplied with a generous amount of the energy and instructions they require to perform tasks, leaving the capability of artificial general intelligence far from being realized. When conceptualizing the perspective of a machine and how a machine would choose to exist, it may be possible that a machine would find it more convenient to exist in outer-space, where solar energy is available in abundance, friction would be lesser, the machine could perform independent research to determine more about the properties and composition of the universe, source raw materials from space [128] and plan missions that span billions of years. Independent, artificially-conscious machines may be least interested in domination over humans, and the technology could very well form the basis for creating multiple small machines that cooperate to form larger bodies capable of exploring and understanding more about the universe. It could also go the other way around, where machines build smaller machines which continue building even smaller machines.

\section{INFERENCES}

In future work, the information from Sect. III can be augmented with more information, categorized and logically analyzed using hypotheses. The process would help narrow 
down on the core aspects of intelligence. For now, a few basic inferences are attempted.

\section{A. Objective}

It is important not to lose sight of what mankind seeks to achieve. The objective of creating an AI is not to imitate the human brain or to imitate biological life. The objective is to create machines that are capable of intelligence that is as good or better than human understanding and intelligence. It should also not be forgotten that human capabilities have evolved from millions of years of evolution (and oddly, we do not know a lot about how our own body works). Facts from Table II and Sects. [II-B1, III-C2 and III-C3 prove that technology can be developed using properties of any available material, while the intelligence and understanding generated thus, can be specific to the technology that created it. The progress of AI has been impeded by the limitations of technology mentioned in Sect. III-F If intelligence cannot be defined yet, the least that can be done is to obtain clarity on the objectives that can be pursued, based on the knowledge from Sect. III.

\section{B. Obtaining clarity about AI}

Is it too early to make conclusions about intelligence? : Yes. Sects. III-A and III-C show that there is a lot more to discover about the properties of molecules and cooperative behavior, before making conclusions about intelligence.

Do neurons or DNA have to be mimicked to simulate intelligence? : No. Table II. Sects. III-B1 and III-C2 show that signaling and storage of information can be performed in any manner, using any suitable technology. Sections III-D8 and III-E2 show how intelligence is unaffected by stroke, partial removal of the hippocampus or starvation, but in cases of intellectual impairment, the lack of oxygen or iodine at a young age can impair intelligence lifelong, due to the lack of maturation of the hippocampus [129]. Studies on traumatic brain injury [130] have shown that intelligence is a function of multiple overlapping capabilities of the brain [130]. This means that the breadth and depth of available memories and the degree of associativity among various types of memories play a role in intelligence. A clue into storage mechanisms may be derived from the phenomenon where even something that had been exciting, becomes boring on repetition. Even microorganisms could be capable of intelligence [21]. The design of algorithms that perform deductions using available data and the design of memory storage techniques are crucial to simulate intelligence. Intelligence needs to be supported by an understanding, which is the capacity to fit phenomena to known models.

Does macro-life follow similar patterns as micro-life?: Yes. Cell membranes are like human skin. Cells differentiating into various types, are like humans taking up different professions. Cell signaling is similar to various communication networks humans have. Cells identifying as belonging to a group or body is similar to human communities. There appears to be a pattern that is followed when smaller groups of agents join to form a larger agent [131]. However, as mentioned in Sect. III-A, the rules defining the patterns will always be adapted to the conditions and forces experienced by the larger agent. This also means that it should theoretically be possible to derive inferences about intelligence, by observing the manner in which human communities form a collective intelligence and a purpose-driven existence.

Are mutations, crossovers, aging and death necessary? : Mutations and crossovers are accidents, errors and tweaks happening to biological data. The discovery of x-rays, the spherical shape of the Earth [132] and heliocentrism are few examples of how accidents, errors and lateral thinking can result in better conclusions or discoveries than purely logical thinking. Errors, complications or wastes accumulating in the body or in memory, does necessitate either a cleanup or death. However, the Banyan, Pando and Turritopsis dohrnii mentioned in Sect. III-C3 and Table I. show that mutations, crossovers, aging and death are not an essential part of life, but are useful features under certain circumstances. Artificial intelligence can therefore be conceptualized as possessing the capability of performing experimental body-modifications, replication and essential-memory summarization which gets passed on to a new or extended body.

Should AI be built with multiple senses?: Yes. Even simple applications can benefit from multiple senses, as shown by Sect. III-D. For example, a simple speech-processing application would require the capability of producing sound on its own (not just by using software, but also with physical actuators), comparing those sounds with the audio inputs received and stored by it, long-term event-based memory and spatial senses to understand Doppler effect or the characteristics of the audio source. A more complex computer vision application for a self driving car for example, would require pixel information, fuel/battery information, tactile inputs (from all surfaces including the tires), spatial inputs, perception of time, knowledge of who humans are, knowledge of why humans travel from one point to another, the consequences of collision, expenses incurred, how the expenses affect the human and the vehicle, consequences of crashing into an obstacle and why the consequences are detrimental. These are the realities of what makes humans knowledgeable enough to drive a vehicle. Such depth and breadth of knowledge is a crucial part of designing any AI that is meant to perform complex tasks.

Does all sensory information need to be combined?: Sometimes yes, sometimes no. Musical memory, the Molyneux problem, stuttering, the Gestalt principles, etcetera in Sect. III-D show that a selective combination of relevant senses would work just fine. However, this combination of senses would benefit from the creation of a temporary world model that represents the current spatial and situational context the agent currently exists in. Place cells [133] are one example of this concept.

Should AI have a body and a face?: A strong AI definitely requires a body that helps it explore and experience the real world. To fill gaps in missing sensory information, an AI needs to use stored inputs to infer a world model and also be capable of using existing information to build its own world model, as evidenced in the anomalies of Sect. III-E4 Communication of information between agents happens via 
body-language, pheromones and multiple other mechanisms. As long as communication happens well, a face is not necessary. Features should be designed based on functionality, rather than mimicking biological features for the sake of familiarity.

Is current technology sufficient for strong AI?: A Turingcomplete computing machine should theoretically be capable of strong AI if the appropriate algorithms, abstraction techniques and memory storage techniques are innovated. However, a technology that possesses fundamental primitives with the capability of accounting for the multi-dimensional combinatorial possibilities of the real-world and is more flexible than current mathematical techniques, would be more efficient and resilient. Qubits of quantum computing may also hold promise in this field.

Are attention-span, emotion and feelings required?: Attention spans are necessary to focus on tasks. Emotions are a concept that is not fully understood, but since they may be a method of retaining attention span for significant events based on the consequences of the events, emotions could potentially be designed as attention-spans that are tied to event consequences. Emotion also needs to account for a sense of belonging to a community, an extension of self into the community and even inanimate objects (similar to how a tool or vehicle is used as an extended capability of the body). However it is a puzzle as to why we "feel" that some things beautiful and why we need to execute bodily actions in certain ways when laughing, crying, etc.

Are free-will and consciousness required?: A weak AI does not require it, as it is meant to perform pre-defined tasks to perfection. A strong AI requires a temporary set of goals created, deleted and updated by itself, to simulate free-will (which offers resilience in handling unpredictable situations), but until a clear understanding of consciousness is obtained, consciousness should at a minimum be simulated via the recognition of body extents, goals, time perception, own capabilities and a recognition of the capabilities of other living and non-living agents.

How should memories be stored? : Certain AI's would require extremely accurate memory storage, while AI's dealing with multi-dimensional real-world information would benefit from storing incrementally complex information, almost similar to how a CNN stores information in layers. Section III-D7 mentions that memory encoding and recall is not fully understood, but it is important to design functionality based on specific needs and available technology, rather than assuming that biological techniques are superior or better-suited for a use-case. Any AI would benefit from a memory designed to quickly access a lifetime of experiences multiple times even for trivial decision-making tasks. This paper proposes that memory be designed as events, since even commonsense is a result of consulting vast stores of memories and experiences, rather than a mere utilization of simplistic, symbolic rules.

Is intelligence a function of community? : Not entirely, but the exchange of information and validation from multiple agents does appear to enhance intelligence, cooperation and submissiveness to agents with higher knowledge (which allows the leading agent to utilize servitude to pursue larger goals, obtain a wider perspective and achieve greater intelligence).
Intelligence appears to be more complex than a mere processing of instructions. Communication and the influence of microscopic and macroscopic sources also appears to contribute to the complex dimension of intelligence. Section III-E3 lists such experiments, while Table I and Sect. III-C4 list multiple capabilities where errors, experimentation, observation, division of labor and communication helped biological organisms escape biases and local optima, to evolve improved technology and understanding.

Is simulating experiences like pain necessary? : Perhaps. The reason why pain is painful is not fully understood [134], although there is an obvious benefit to create a notification system of damages to any physical body-part or even to software (damages caused by malware or untimely malfunctions).

Can current AI be called AI?: No. Ideally, current stateof-the-art can at-best be called an Analytical Engine or Machine Learning. Machine Intelligence or Artificial Intelligence requires more advanced capabilities to be identified as intelligence, even if it does not resemble animal intelligence.

What goals should an AI pursue?: If a living organism were guaranteed a constant supply of nutrition, safety and elimination of wastes, it would have no incentive to do anything else. An agent in a dormant state (a virus, a seed or worms in natural cryopreservation [135]), could remain inactive indefinitely. The goals of biological agents appear to be a derivative of needs. The survival instinct, dormancy and replication appear to have arisen from the need to preserve the means and knowhow of creating the chemical equilibrium of life. Mutations, selfish-DNA, gametes and plasmids (Sects. III-C3 and III-C4) appear to be created from a need to improve and consolidate important knowledge. Every action and decision is imposed by internal or external stimuli. Machines on the other hand, are different. As long as machines are supplied with electricity and can malfunction without negative consequences to itself, a machine will have no incentive to do anything that drives it to acquire intelligence. Therefore, goals can be defined based on the types of AI:

1) Weak AI's would perform specific, human-defined tasks.

2) Assistive AI's built as an embodied consciousness can have goals of utilizing a lifetime of memories and sensory inputs to understand the function and purpose of everything it encounters and understand the consequences of events, thus being capable of performing the core tasks it is assigned, create temporary goals to achieve intermediate tasks, learn and explore new phenomena once the core task is completed, make use of other objects or agents to accomplish tasks, follow orders, be capable of sourcing energy on its own and repairing itself using pre-built parts or by manufacturing them. Such an AI could potentially learn enough to be a threat to humanity, but regulations, surveillance and the AI's dependency on raw materials prevents such an eventuality.

3) Strong AI's can possess consciousness, curiosity, general-intelligence and the capability to invent. Building such an AI is not a necessity, but from all of Sect. III. a fundamental goal of living creatures that stands 
out, is the pursuit of better knowledge. A machine or a human-augmented machine that can explore the universe, build larger machines as super-sets of smaller cooperative machines, discover more about the universe, and constantly translate and share the knowledge with humanity, would perhaps satisfy the deepest thirst of mankind: Overcoming physical limitations of the body and understanding more about the universe to eventually find out why we exist.

\section{CONClusion}

This paper seeks to highlight three important points:

1) It is not necessary to mimic nature to create an AI. It is more important to identify what has to be achieved, and use or invent suitable technology that will shape AI according to the need.

2) Human behavior and human communities follow similar patterns as microscopic life, thus offering similar clues at an easier-to-observe, macroscopic scale (although studying microscopic phenomena is useful when investigating the origin of any concept).

3) Intelligent decisions require consulting vast stores of memories (experience) even for trivial tasks. To achieve this, memories are perhaps best stored as events.

A few centuries ago, nobody could predict the invention of software. Similarly, the future may hold technologies and AI capabilities that are far beyond the comprehension of contemporary researchers. To achieve such progress, it is necessary for a small section of academia to continue actively encouraging interdisciplinary collaboration between scientific departments to pursue alternate ideas and discover new technologies, regardless of funding or commercial interests. Information in Sects. III and IV show how a concise but broad perspective of facts and anomalies can help in identifying similar patterns, which simplifies the process of logically eliminating incorrect assumptions about the technology and goals of AI. Simplistic representation of intelligence in mathematical and software models are sufficient for machine learning or weak AI. However, rather than strive to implement conscious intelligence via a strong AI capable of artificial general intelligence, it is more appropriate to recognize that the purpose of building AI was to create cost-effective assistants (or even loyal companions). Hardware, algorithms, cognitive architectures and theories on intelligence need to be re-visited and re-built from the groundup, since intelligence is a function of events, consequences, collaboration, tweaks, errors, error-correction, observation and logical analysis of various types of information. The perspective offered via this paper hopes to have assisted the reader zoom from the level of the universe, down to the level of biological nano-machines and the vast complexities of life, matter and unknown forces, thus providing a quickreference when designing the technologies that approach our understanding of intelligence. This paper is also an ode to the toil and sacrifices of every researcher and philosopher on whose shoulders we stand today.

\section{REFERENCES}

[1] T. Browne, Pseudodoxia epidemica, or, Enquiries into very many received tenents, and commonly presumed truths. E. Dod, 1978.

[2] R. J. Sternberg, Handbook of intelligence. Cambridge University Press, 2000.

[3] D. Crevier, AI: the tumultuous history of the search for artificial intelligence. Basic Books, Inc., 1993.

[4] H. Moravec, Mind children: The future of robot and human intelligence. Harvard University Press, 1988.

[5] P. McCorduck and C. Cfe, Machines who think: A personal inquiry into the history and prospects of artificial intelligence. CRC Press, 2004.

[6] A. Kaplan and M. Haenlein, "Siri, siri, in my hand: Who's the fairest in the land? on the interpretations, illustrations, and implications of artificial intelligence," Business Horizons, vol. 62, no. 1, pp. 15-25, 2019.

[7] E. Schrödinger, What is life?: the physical aspect of the living cell, and, Mind and matter. Cambridge University Press, 1967.

[8] R. A. Brooks, "Elephants don't play chess," Robotics and autonomous systems, vol. 6, no. 1-2, pp. 3-15, 1990.

[9] M. Kiefer and N. M. Trumpp, "Embodiment theory and education: The foundations of cognition in perception and action," Trends in Neuroscience and Education, vol. 1, no. 1, pp. 15-20, 2012.

[10] R. R. Souza, "Algorithms, future and digital rights: Some reflections," Education for Information, vol. 34, no. 3, pp. 179-183, 2018.

[11] A. Sloman, "The mythical turing test," Alan Turing: His work and impact, pp. 606-611, 2013.

[12] J. Hernandez-Orallo, "Ai evaluation: On broken yardsticks and measurement scales," 2020.

[13] J. Hawkins and S. Blakeslee, On intelligence: How a new understanding of the brain will lead to the creation of truly intelligent machines. Macmillan, 2007.

[14] R. Granger, S. Petrovic, A. Felch, J. Kerr, M. Johnson, C. Wuerth, and J. Benvenuto, "Engines of the brain: the computational "instruction set" of perception and cognition," in AAAI Symposium (N. Cassimatis, Ed), Washington DC, 2005.

[15] N. A. Goriounova and H. D. Mansvelder, "Genes, cells and brain areas of intelligence," Frontiers in human neuroscience, vol. 13, 2019.

[16] T. F. Glick, S. Livesey, and F. Wallis, Medieval science, technology, and medicine: an encyclopedia. Routledge, 2014, vol. 11.

[17] C. Scharf, The Zoomable Universe: An Epic Tour Through Cosmic Scale, from Almost Everything to Nearly Nothing. Scientific American/Farrar, Straus and Giroux, 2017.

[18] M. B. Cilek, "Redefining gravity: Field versus flow," Private Fundamental Research. Istanbul. gravity@ superonline.com, relativity@ superonline. com, 2017.

[19] D. B. Dusenbery, Living at micro scale: the unexpected physics of being small. Harvard University Press, 2009.

[20] G. F. Ellis and G. Brundrit, "Life in the infinite universe," Quarterly Journal of the Royal Astronomical Society, vol. 20, pp. 37-41, 1979.

[21] B. Ford, "Are cells ingenious?" Microscope-Chicago, vol. 52, no. 3-4, pp. 135-144, 2004.

[22] S. Mitoh and Y. Yusa, "Extreme autotomy and whole-body regeneration in photosynthetic sea slugs," Current Biology, vol. 31, no. 5, pp. R233R234, 2021.

[23] H. Safavi-Hemami, J. Gajewiak, S. Karanth, S. D. Robinson, B. Ueberheide, A. D. Douglass, A. Schlegel, J. S. Imperial, M. Watkins, P. K. Bandyopadhyay et al., "Specialized insulin is used for chemical warfare by fish-hunting cone snails," Proceedings of the National Academy of Sciences, vol. 112, no. 6, pp. 1743-1748, 2015.

[24] W. Wesołowska and T. Wesołowski, "Do 1 eucochloridium sporocysts manipulate the behaviour of their snail hosts?" Journal of Zoology, vol. 292, no. 3, pp. 151-155, 2014.

[25] G. M. del Marmol, O. Mozaffari, and J. Gállego, "Pseudocerastes urarachnoides: the ambush specialist," waterbirds, vol. 10, pp. 117126, 2016.

[26] P. Govind, "Overviews on diversity of fish," Research Journal of Animal, Veterinary and Fishery Science, vol. 1, pp. 12-18, 2013.

[27] J. Sun, C. Chen, N. Miyamoto, R. Li, J. D. Sigwart, T. Xu, Y. Sun, W. C. Wong, J. C. Ip, W. Zhang et al., "The scaly-foot snail genome and implications for the origins of biomineralised armour," Nature communications, vol. 11, no. 1, pp. 1-12, 2020.

[28] S. Verma, A. Johnson, H. Haile, and S. Reinsch, "How to kill a tardigrade-without even trying," 2018. 
[29] S. P. Mahal, S. Browning, J. Li, I. Suponitsky-Kroyter, and C. Weissmann, "Transfer of a prion strain to different hosts leads to emergence of strain variants," Proceedings of the National Academy of Sciences, vol. 107, no. 52, pp. 22 653-22 658, 2010.

[30] K. Grünberg, E.-C. Müller, A. Otto, R. Reszka, D. Linder, M. Kube, R. Reinhardt, and D. Schüler, "Biochemical and proteomic analysis of the magnetosome membrane in magnetospirillum gryphiswaldense," Appl. Environ. Microbiol., vol. 70, no. 2, pp. 1040-1050, 2004.

[31] Y. Gong, D. Patterson, Y. Li, Z. Hu, M. Sommerfeld, Y. Chen, and Q. Hu, "Vernalophrys algivore gen. nov., sp. nov," Rhizaria: Cercozoa: Vampyrellida), a new, 2015.

[32] J. M. Tiedje, "Shewanella - the environmentally versatile genome," Nature biotechnology, vol. 20, no. 11, pp. 1093-1094, 2002

[33] K. Zahradka, D. Slade, A. Bailone, S. Sommer, D. Averbeck, M. Petranovic, A. B. Lindner, and M. Radman, "Reassembly of shattered chromosomes in deinococcus radiodurans," Nature, vol. 443, no. 7111, pp. 569-573, 2006.

[34] J. Flegr and A. Markoš, "Masterpiece of epigenetic engineering-how toxoplasma gondii reprogrammes host brains to change fear to sexual attraction," Molecular ecology, vol. 23, no. 24, pp. 5934-5936, 2014.

[35] M. Kramar and K. Alim, "Encoding memory in tube diameter hierarchy of living flow network," Proceedings of the National Academy of Sciences, vol. 118, no. 10, 2021.

[36] D. Vogel and A. Dussutour, "Direct transfer of learned behaviour via cell fusion in non-neural organisms," Proceedings of the Royal Society B: Biological Sciences, vol. 283, no. 1845, p. 20162382, 2016.

[37] J. Taylor and S. Patek, "Ritualized fighting and biological armor: the impact mechanics of the mantis shrimp's telson," Journal of Experimental Biology, vol. 213, no. 20, pp. 3496-3504, 2010.

[38] S. Korenko, J. Smerda, and S. Pekar, "Life-history of the parthenogenetic oonopid spider, triaeris stenaspis (araneae: Oonopidae)," european Journal of entomology, vol. 106, no. 2, pp. 217-223, 2009.

[39] J. B. Swann, S. J. Holland, M. Petersen, T. W. Pietsch, and T. Boehm, "The immunogenetics of sexual parasitism," Science, vol. 369, no. 6511, pp. 1608-1615, 2020

[40] D. J. Larson, L. Middle, H. Vu, W. Zhang, A. S. Serianni, J. Duman, and B. M. Barnes, "Wood frog adaptations to overwintering in alaska: new limits to freezing tolerance," Journal of Experimental Biology, vol. 217, no. 12, pp. 2193-2200, 2014.

[41] C. D. de Santana, W. G. Crampton, C. B. Dillman, R. G. Frederico, M. H. Sabaj, R. Covain, J. Ready, J. Zuanon, R. R. de Oliveira, R. N. Mendes-Júnior et al., "Unexpected species diversity in electric eels with a description of the strongest living bioelectricity generator," Nature communications, vol. 10, no. 1, pp. 1-10, 2019.

[42] J. Li, S. Browning, S. P. Mahal, A. M. Oelschlegel, and C. Weissmann, "Darwinian evolution of prions in cell culture," Science, vol. 327, no. 5967, pp. 869-872, 2010.

[43] C. W. Dunn, S. P. Leys, and S. H. Haddock, "The hidden biology of sponges and ctenophores," Trends in ecology \& evolution, vol. 30, no. 5, pp. 282-291, 2015.

[44] J. Levine, "Materialism and qualia: The explanatory gap," Pacific philosophical quarterly, vol. 64, no. 4, pp. 354-361, 1983.

[45] B. Calcott and K. Sterelny, "The major transitions in evolution revisited," 2011.

[46] B. Alberts, A. Johnson, J. Lewis, M. Raff, K. Roberts, and P. Walter, "Drosophila and the molecular genetics of pattern formation: Genesis of the body plan," in Molecular Biology of the Cell. 4th edition. Garland Science, 2002.

[47] L. Pray, "Dna replication and causes of mutation," Nature education, vol. 1, no. 1, p. 214, 2008.

[48] W. Y. Timothy and C. I. Bargmann, "Dynamic regulation of axon guidance," Nature neuroscience, vol. 4, no. 11, pp. 1169-1176, 2001.

[49] V. Merrill, R. Diederich, F. Turner, and T. Kaufman, "A genetic and developmental analysis of mutations in labial, a gene necessary for proper head formation in drosophila melanogaster," Developmental biology, vol. 135, no. 2, pp. 376-391, 1989.

[50] V. Brinkmann, U. Reichard, C. Goosmann, B. Fauler, Y. Uhlemann, D. S. Weiss, Y. Weinrauch, and A. Zychlinsky, "Neutrophil extracellular traps kill bacteria," science, vol. 303, no. 5663, pp. 1532-1535, 2004.

[51] J. Ling, P. O'donoghue, and D. Söll, "Genetic code flexibility in microorganisms: novel mechanisms and impact on physiology," Nature Reviews Microbiology, vol. 13, no. 11, pp. 707-721, 2015.

[52] M. El Karoui, M. Hoyos-Flight, and L. Fletcher, "Future trends in synthetic biology-a report," Frontiers in bioengineering and biotechnology, vol. 7, p. 175, 2019.
[53] E. Bearer and P. Satpute-Krishnan, "The role of the cytoskeleton in the life cycle of viruses and intracellular bacteria: tracks, motors, and polymerization machines," Current Drug Targets-Infectious Disorders, vol. 2, no. 3, pp. 247-264, 2002.

[54] A. Karami, "Largest and smallest genome in the world."

[55] J. DeWoody, C. A. Rowe, V. D. Hipkins, and K. E. Mock, "”pando" lives: molecular genetic evidence of a giant aspen clone in central utah," Western North American Naturalist, vol. 68, no. 4, pp. 493-497, 2008

[56] L. S. Frost, R. Leplae, A. O. Summers, and A. Toussaint, "Mobile genetic elements: the agents of open source evolution," Nature Reviews Microbiology, vol. 3, no. 9, pp. 722-732, 2005.

[57] M. Frechin, T. Stoeger, S. Daetwyler, C. Gehin, N. Battich, E.-M Damm, L. Stergiou, H. Riezman, and L. Pelkmans, "Cell-intrinsic adaptation of lipid composition to local crowding drives social behaviour," Nature, vol. 523, no. 7558, pp. 88-91, 2015.

[58] N. F. Aykan, Intercellular Communication. ISBN, 2012.

[59] M. B. Clarke and V. Sperandio, "Events at the host-microbial interface of the gastrointestinal tract iii. cell-to-cell signaling among microbial flora, host, and pathogens: there is a whole lot of talking going on," American Journal of Physiology-Gastrointestinal and Liver Physiology, vol. 288, no. 6, pp. G1105-G1109, 2005.

[60] J.-H. Mao, Y.-M. Kim, Y.-X. Zhou, D. Hu, C. Zhong, H. Chang, C. Brislawn, S. Langley, Y. Wang, B. L. Peisl et al., "Genetic and metabolic links between the murine microbiome and memory," Microbiome, vol. 8, pp. 1-14, 2020.

[61] T. J. A. Craddock and J. A. Tuszynski, "A critical assessment of the information processing capabilities of neuronal microtubules using coherent excitations," Journal of biological physics, vol. 36, no. 1, p. 53, 2010.

[62] F. Kracke, I. Vassilev, and J. O. Krömer, "Microbial electron transport and energy conservation-the foundation for optimizing bioelectrochemical systems," Frontiers in microbiology, vol. 6, p. 575, 2015.

[63] T. Chang, "Hemoperfusion based on artificial cells for aluminium and iron removal, immunosorption, fulminant hepatic failure, uremia, poisoning and metabolic assists," The International journal of artificial organs, vol. 9, no. 5, pp. 285-288, 1986.

[64] J. Gsponer, M. E. Futschik, S. A. Teichmann, and M. M. Babu, "Tight regulation of unstructured proteins: from transcript synthesis to protein degradation," Science, vol. 322, no. 5906, pp. 1365-1368, 2008.

[65] F. Baluška and S. Mancuso, "Vision in plants via plant-specific ocelli?" Trends in plant science, vol. 21, no. 9, pp. 727-730, 2016.

[66] C. López-Otín, M. A. Blasco, L. Partridge, M. Serrano, and G. Kroemer, "The hallmarks of aging," Cell, vol. 153, no. 6, pp. 1194-1217, 2013.

[67] F. Jackson, "Epiphenomenal qualia," The Philosophical Quarterly (1950-), vol. 32, no. 127, pp. 127-136, 1982.

[68] J. D. Blom and I. E. Sommer, "Auditory hallucinations: nomenclature and classification," Cognitive and behavioral neurology, vol. 23, no. 1 , pp. 55-62, 2010

[69] T. A. Stroffregen and J. B. Pittenger, "Human echolocation as a basic form of perception and action," Ecological psychology, vol. 7, no. 3, pp. 181-216, 1995.

[70] A. J. Blood and R. J. Zatorre, "Intensely pleasurable responses to music correlate with activity in brain regions implicated in reward and emotion," Proceedings of the National Academy of Sciences, vol. 98, no. 20, pp. $11818-11823,2001$.

[71] R. L. Gregory and J. G. Wallace, "Recovery from early blindness," Experimental psychology society monograph, vol. 2, pp. 65-129, 1963.

[72] C. Ackroyd, N. Humphrey, and E. K. Warrington, "Lasting effects of early blindness a case study," Quarterly Journal of Experimental Psychology, vol. 26, no. 1, pp. 114-124, 1974.

[73] D. M. Eagleman, "Visual illusions and neurobiology," Nature Reviews Neuroscience, vol. 2, no. 12, pp. 920-926, 2001.

[74] K. Koffka, Principles of Gestalt psychology. Routledge, 2013, vol. 44

[75] Q. Li and C. Montell, "Mechanism for food texture preference based on grittiness," Current Biology, 2021.

[76] N. Gosselin, I. Peretz, E. Johnsen, and R. Adolphs, "Amygdala damage impairs emotion recognition from music," Neuropsychologia, vol. 45, no. 2, pp. 236-244, 2007.

[77] C. Finke, N. E. Esfahani, and C. J. Ploner, "Preservation of musical memory in an amnesic professional cellist," Current Biology, vol. 22, no. 15, pp. R591-R592, 2012.

[78] D. J. Kraemer, C. N. Macrae, A. E. Green, and W. M. Kelley, "Sound of silence activates auditory cortex," Nature, vol. 434, no. 7030, pp. 158-158, 2005. 
[79] G. Robles-De-La-Torre, "The importance of the sense of touch in virtual and real environments," Ieee Multimedia, vol. 13, no. 3, pp. 24-30, 2006.

[80] A. Amedi, L. B. Merabet, J. Camprodon, F. Bermpohl, S. Fox, I. Ronen, D.-S. Kim, and A. Pascual-Leone, "Neural and behavioral correlates of drawing in an early blind painter: a case study," Brain research, vol. 1242, pp. 252-262, 2008

[81] K. Sanematsu, M. Kitagawa, R. Yoshida, S. Nirasawa, N. Shigemura, and Y. Ninomiya, "Intracellular acidification is required for full activation of the sweet taste receptor by miraculin," Scientific reports, vol. 6 , p. $22807,2016$.

[82] J. M. Boyce and G. Shone, "Effects of ageing on smell and taste," Postgraduate medical journal, vol. 82, no. 966, pp. 239-241, 2006.

[83] A. R. Nath and M. S. Beauchamp, "A neural basis for interindividual differences in the mcgurk effect, a multisensory speech illusion," Neuroimage, vol. 59, no. 1, pp. 781-787, 2012.

[84] J. Willis and A. Todorov, "First impressions: Making up your mind after a 100-ms exposure to a face," Psychological science, vol. 17, no. 7, pp. 592-598, 2006.

[85] R. Rosen, "Pareidolia: A bizarre bug of the human mind emerges in computers," The Atlantic Monthly, vol. 7, 2012.

[86] S. Gilad-Gutnick, E. S. Harmatz, K. Tsourides, G. Yovel, and P. Sinha, "Recognizing facial slivers," Journal of cognitive neuroscience, vol. 30 no. 7, pp. 951-962, 2018.

[87] E. N. Marieb and K. Hoehn, Human anatomy \& physiology. Pearson education, 2007

[88] S. M. Rao, A. R. Mayer, and D. L. Harrington, "The evolution of brain activation during temporal processing," Nature neuroscience, vol. 4, no. 3 , pp. 317-323, 2001

[89] M. A. Changizi, The brain from 25,000 feet: High level explorations of brain complexity, perception, induction and vagueness. Springer Science \& Business Media, 2013, vol. 317.

[90] E. J. Jaldow, D. A. Oakley, and G. C. Davey, "Performance of decorticated rats on fixed interval and fixed time schedules," European Journal of Neuroscience, vol. 1, no. 5, pp. 461-470, 1989.

[91] Z. Atakan, P. Morrison, M. G Bossong, R. Martin-Santos, and J. A Crippa, "The effect of cannabis on perception of time: a critical review," Current pharmaceutical design, vol. 18, no. 32, pp. 49154922, 2012.

[92] L. R. Squire, "Memory and brain systems: 1969-2009," Journal of Neuroscience, vol. 29, no. 41, pp. 12711-12 716, 2009.

[93] E. I. Moser, E. Kropff, and M.-B. Moser, "Place cells, grid cells, and the brain's spatial representation system," Аппи. Rev. Neurosci., vol. 31, pp. 69-89, 2008.

[94] D. J. Daegling, "Functional morphology of the human chin," Evolutionary Anthropology: Issues, News, and Reviews, vol. 1, no. 5, pp. 170-177, 1993.

[95] S. K. Daniels, K. Brailey, D. H. Priestly, L. R. Herrington, L. A Weisberg, and A. L. Foundas, "Aspiration in patients with acute stroke," Archives of physical medicine and rehabilitation, vol. 79, no. 1, pp. 14-19, 1998.

[96] M. T. Orne and K. E. Scheibe, "The contribution of nondeprivation factors in the production of sensory deprivation effects: The psychology of the" panic button."," The Journal of Abnormal and Social Psychology, vol. 68 , no. 1, p. $3,1964$.

[97] M. Hallett, "Volitional control of movement: the physiology of free will," Clinical Neurophysiology, vol. 118, no. 6, pp. 1179-1192, 2007.

[98] S. E. MacPherson and S. Della Sala, Cases of Amnesia: Contributions to Understanding Memory and the Brain. Routledge, 2019.

[99] R. Jacobson, "The persistence of memory," Scientific American, vol. 312, no. 4, pp. 14-16, 2015.

[100] M. Macmillan, "Phineas gage: A case for all reasons," Classic cases in neuropsychology, pp. 220-238, 1996.

[101] M. Bentivoglio and G. Grassi-Zucconi, "The pioneering experimental studies on sleep deprivation," Sleep, vol. 20, no. 7, pp. 570-576, 1997.

[102] N. C. Rattenborg, S. L. Lima, and C. J. Amlaner, "Half-awake to the risk of predation," Nature, vol. 397, no. 6718, pp. 397-398, 1999.

[103] G. W. Williams, "Highway hypnosis: An hypothesis," International Journal of Clinical and Experimental Hypnosis, vol. 11, no. 3, pp. 143-151, 1963.

[104] M.-C. Cammaerts Tricot and R. Cammaerts, "Are ants (hymenoptera, formicidae) capable of self recognition?" Journal of Science, vol. 5, no. 7, pp. 521-532, 2015.

[105] C. Heyes, "Where do mirror neurons come from?" Neuroscience \& Biobehavioral Reviews, vol. 34, no. 4, pp. 575-583, 2010

[106] J. W. Papez, "A proposed mechanism of emotion," Archives of Neurology \& Psychiatry, vol. 38, no. 4, pp. 725-743, 1937.
[107] E. Harmon-Jones, K. Vaughn-Scott, S. Mohr, J. Sigelman, and C. Harmon-Jones, "The effect of manipulated sympathy and anger on left and right frontal cortical activity." Emotion, vol. 4, no. 1, p. 95, 2004.

[108] B. S. Hutauruk, "Children first language acquisition at age 1-3 years old in balata," IOSR Journal of Humanities and Social Science (IOSRJHSS), vol. 20, no. 8, pp. 51-57, 2015.

[109] L. L. LaPointe, "Feral children," Journal of medical speech-language pathology, vol. 13, no. 1, pp. vii-vii, 2005.

[110] J. Smogorzewska, G. Szumski, and P. Grygiel, "Same or different? theory of mind among children with and without disabilities," PloS one, vol. 13, no. 10, 2018.

[111] I. So, "Cognitive development in children: Piaget development and learning," JOURNAL, OF RESEARCH IN SCIENCE TEACHING, vol. 2, pp. 176-186, 1964.

[112] A. Keys, J. Brožek, A. Henschel, O. Mickelsen, and H. L. Taylor, "The biology of human starvation.(2 vols)." 1950.

[113] K. S. Larsen, "Conformity in the asch experiment," The Journal of Social Psychology, vol. 94, no. 2, pp. 303-304, 1974.

[114] T. Blass, Obedience to authority: Current perspectives on the Milgram paradigm. Psychology Press, 1999.

[115] P. G. Zimbardo, "On the ethics of intervention in human psychological research: With special reference to the stanford prison experiment," Cognition, vol. 2, 2017.

[116] R. Raouf, K. Quick, and J. N. Wood, "Pain as a channelopathy," The Journal of clinical investigation, vol. 120, no. 11, pp. 3745-3752, 2010

[117] A. Funkhouser and M. Schredl, "The frequency of déjà vu (déjà rêve) and the effects of age, dream recall frequency and personality factors," International Journal of Dream Research, vol. 3, no. 1, pp. 60-64, 2010

[118] G. P. Prigatano and D. L. Schacter, Awareness of deficit after brain injury: Clinical and theoretical issues. Oxford University Press, 1991.

[119] R. S. Nickerson and M. J. Adams, "Long-term memory for a common object," Cognitive psychology, vol. 11, no. 3, pp. 287-307, 1979.

[120] J. Jordania, "Who asked the first question," The origins of human choral singing, intelligence, language and speech. Tblisi, Georgia: Logos, 2006.

[121] P. Wright and D. Terrett, "A hybrid computer as a training simulator," Radio and Electronic Engineer, vol. 28, no. 4, pp. 261-264, 1964.

[122] L. Gyongyosi and S. Imre, "A survey on quantum computing technology," Computer Science Review, vol. 31, pp. 51-71, 2019.

[123] R. A. Wilson and F. C. Keil, The MIT encyclopedia of the cognitive sciences. MIT press, 2001.

[124] J. McCaskill, "Polymer chemistry on tape: A computational model for emergent genetics." 1988.

[125] I. Budin and N. K. Devaraj, "Membrane assembly driven by a biomimetic coupling reaction," Journal of the American Chemical Society, vol. 134, no. 2, pp. 751-753, 2012.

[126] C. Mead, "Neuromorphic electronic systems," Proceedings of the IEEE, vol. 78 , no. 10 , pp. 1629-1636, 1990.

[127] I. Kotseruba, O. J. A. Gonzalez, and J. K. Tsotsos, "A review of 40 years of cognitive architecture research: Focus on perception, attention, learning and applications," arXiv preprint arXiv:1610.08602, pp. 1-74, 2016.

[128] O. Pike, F. Mackenroth, E. Hill, and S. Rose, "A photon-photon collider in a vacuum hohlraum," Nature Photonics, vol. 8, no. 6, pp. 434-436, 2014.

[129] E. McClendon, K. Wang, K. Degener-O'Brien, M. W. Hagen, X. Gong, T. Nguyen, W. W. Wu, J. Maylie, and S. A. Back, "Transient hypoxemia disrupts anatomical and functional maturation of preterm fetal ovine ca1 pyramidal neurons," Journal of Neuroscience, vol. 39, no. 40, pp. 7853-7871, 2019

[130] A. K. Barbey, R. Colom, J. Solomon, F. Krueger, C. Forbes, and J. Grafman, "An integrative architecture for general intelligence and executive function revealed by lesion mapping," Brain, vol. 135, no. 4 , pp. 1154-1164, 2012.

[131] N. Hughes-Jones, "Inter-group aggression: The multi-individual organism and the survival instinct," Medicine, Conflict and Survival, vol. 16 , no. 2 , pp. $231-235,2000$

[132] I. Asimov, How did we find out the earth is round? White Lion Publishers, 1973

[133] J. O'Keefe and J. Dostrovsky, "The hippocampus as a spatial map: Preliminary evidence from unit activity in the freely-moving rat." Brain research, 1971.

[134] G. Corder, B. Ahanonu, B. F. Grewe, D. Wang, M. J. Schnitzer, and G. Scherrer, "An amygdalar neural ensemble that encodes the 
unpleasantness of pain," Science, vol. 363, no. 6424, pp. 276-281, 2019.

[135] A. Shatilovich, A. Tchesunov, T. Neretina, I. Grabarnik, S. Gubin, T. Vishnivetskaya, T. C. Onstott, and E. Rivkina, "Viable nematodes from late pleistocene permafrost of the kolyma river lowland," in Doklady Biological Sciences, vol. 480, no. 1. Springer, 2018, pp. 100-102. 